\title{
Hacia un desarrollo profesional convergente de la orientación escolar
}

\section{Francisco Javier Peralta Sánchez}

\author{
Orientador Escolar, IES Algazul, Roquetas de Mar (Almería) \\ Presidente de la Asociación Andaluza \\ de Psicología Educativa y Psicopedagogía
}

\section{España}




\section{Resumen}

En este artículo abordamos diferentes aspectos esenciales en el desarrollo profesional del perfil de orientador escolar. En un primer apartado, realizamos un repaso del efecto de la formación universitaria, la miniespecialización durante la preparación de los procesos selectivos (oposiciones), la formación inicial en la profesión, las actividades de co-formación y las de perfeccionamiento en el perfil de la orientación escolar. En un segundo apartado, analizamos el perfil de orientación dentro de la legislación vigente. En tercer lugar, analizamos la percepción que tienen tanto los evaluadores del sistema educativo, como los usuarios directos del mismo, del rol de orientador. Terminamos el artículo con los nuevos retos que podemos plantear en el futuro de la profesión orientadora.

Palabras-clave: orientación escolar, asesoramiento psicopedagógico. 


\section{Introducción}

El concepto de orientación, aunque muy genérico, parece que ha consigo un cierto consenso. Bisquerra (2004; p. 67) lo define como "un proceso de ayuda continuo a todas las personas, en todos los aspectos, con la finalidad de potenciar el desarrollo humano a lo largo de toda la vida". En este sentido hablar de orientación y de educación sería lo mismo. Desde esta premisa orientadores son todos los docentes, pues la tutoría y la orientación de los alumnos es una función inherente a la función docente (LOGSE; art. 60.1). Sin embargo, en este artículo vamos a analizar cómo se engendra y desarrolla profesionalmente el perfil de orientador escolar, como aquel profesional que se especializa en tareas específicas de esta índole y que ejerce su profesión bien en equipos de sector, bien en departamentos de orientación, sabiendo que existen otros colegas, también "orientadores" que desempeñan sus tareas en otros organismos fuera de la enseñanza (oficinas de búsqueda de empleo, selección de recursos humanos, servicios de salud, servicios de orientación para profesionales del ejército, etc.).

A partir de aquí, se podría pensar, tal vez, salvando los matices derivados del análisis de necesidades del lugar en concreto donde ejerce su profesión, que el modo y los principios de actuación en orientación escolar deberían ser los mismos. Pero la práctica profesional hace que nos interroguemos acerca de variaciones que intuimos que pueden acaecer, así como de otras variables relacionadas con esta temática. Por ello, nos preguntamos: ¿Es homogéneo el modo de actuación de los distintos profesionales de la orientación en las áreas, centros y etapas educativas en las que intervienen? ¿Qué variables intervienen en el perfil del puesto de orientador? ¿De qué depende su evolución y trayectoria profesional desde que se incorpora por primera vez a un centro educativo hasta que promocionan a otros escalafones profesionales o procede a la jubilación?

Las repuestas a estas preguntas no es cuestión fácil, pues para ello necesitaríamos definir una serie de parámetros e indicadores de eficacia y eficiencia a través de los cuales establecer en qué medida las distintas administraciones educativas (comunidades autónomas) planifican los ámbitos de intervención orientadora y en qué medida los profesionales dan respuesta a lo programado -Planes de Actuación de los Equipos de Sector o Planes de Orientación y Acción Tutorial de los centros escolares-- Paraleamente, deberíamos cotejar cómo los profesionales de la orientación nos ajustamos o no a las expectativas y requerimientos de otros colectivos profesionales (docentes, educadores, logopedas...), con los que podemos tra- 
bajar interdisciplinarmente, e incluso de los alumnos, últimos receptores y beneficiarios de la orientación y de la acción tutorial, además de sus familias.

En este sentido debemos distinguir tres niveles en los que se desarrolla la orientación y la acción tutorial, aunque en este artículo sólo vamos a abordar el desarrollo profesional de los profesionales que intervienen en el segundo y tercer nivel de actuación. Los tres niveles a los que aludimos son estos:

a) Nivel de grupo-clase o tutoría: la función tutorial y orientadora corresponde al equipo educativo que interviene con ese grupo de alumnos y, en especial, al profesor tutor de dicho grupo clase.

b) Nivel de centro educativo: como claustro de profesores con todos los recursos humanos puestos a su disposición. Al menos en los centros de Educación Secundaria se cuenta con el perfil de Departamentos de Orientación.

c) Por el principio de sectorización, las distintas comunidades autónomas, aunque con diferentes denominaciones, cuentan con los Equipos de Sector (Equipos de Orientación Educativa y Psicopedagógica)

El desarrollo profesional del perfil de orientador escolar probablemente estará condicionado, al igual que cualquier otra profesión, por variables personales (motivaciones, expectativas de promoción docente, variables de personalidad. etc.) y variables de índole laboral (clima de trabajo, oportunidades de trabajo en equipo, disponibilidad de recursos, acceso a programas y organismos especializados en formación, incentivos de reciclado profesional, etc.) Pero dentro de toda esta amalgama de variables no podemos ni debemos olvidar otras que tienen un enorme impacto en el desarrollo de la carrera profesional. Nos referimos a las variables de formación inicial (al período previo de preparación y selección para el ejercicio profesional directo) y a las variables de formación permanente y recurrente del profesorado.

Vamos a distinguir, por lo tanto, varios tipos de variables en este apartado:

a) Las variables iniciales destinadas a la obtención del título universitario y profesional que capacite para el ejercicio de la profesión orientadora.

b) Variables previas destinadas a la preparación para la superación de la fase de selección. 
c) Variables de formación permanente y recurrente, una vez que ya se ha entrado o se ha iniciado el ejercicio profesional.

Veamos más pormenorizadamente cada una de estas variables.

\section{Influencia de la formación inicial y del perfeccionamiento en el perfil del orientador es- colar}

Formación académica inicial para la obtención del título universitario

Dentro de este bloque de variables debemos distinguir varios matices, ya que cada una de ellas tendrá sin duda repercusiones en el ejercicio de la orientación. En primer lugar, consideramos que hay un bloque carreras universitarias dentro del campo de la Psicología, Pedagogía o la Psicopedagogía. Los planes de estudios de estas disciplinas humanísticas tratan distintos aspectos que son necesarios para el posterior ejercicio profesional en el campo de la orientación. Pero también existen diferencias notables que van a condicionar la actuación práctica en la orientación y la forma de concebir, interpretar, evaluar y operar sobre la realidad a la que se enfrentan esos profesionales de la orientación.

Indudablemente, disciplinas como la Psicología contribuirán a una interpretación de la conducta humana y a una intervención más acorde con esos principios teóricos que la conforman. Otras disciplinas como la Pedagogía contribuirán a fundamentar el hecho didáctico y los principios de intervención estarán en consonancia con esas teorías. Finalmente, debemos considerar otra disciplina de más reciente aparición en los planes de estudios de las universidades españolas, como es la Psicopedagogía, disciplina que aúna parte de una y otra disciplina enunciadas anteriormente, con el inconveniente de no contar con la máxima especialización de las disciplinas puras y, en ocasiones, del reconocimiento de colegios profesionales, para optar, por ejemplo, a la Relación de Puestos de Trabajo, como orientadres, del SAE (Servicio Andaluz de Empleo).

En cualquier caso tanto disciplinas puras como la Psicología o la Pedagogía, u otras misceláneas, como la Psicopedagogía conforman un marco teórico que debe ser conectado con la realidad práctica profesional. De ahí que después de esa preparación general, encaminada para ejercer distintos perfiles profesionales y no solo el de orientador, esas titulaciones 
deban de contribuir para formar a los futuros profesionales en función de su ámbito de actuación.

Pararnos a contemplar en qué medida estas titulaciones contribuyen en concreto a la formación del rol del fututo orientador nos llevaría a realizar un análisis de los Planes de estudios de las distintas universidades y a analizar, con efectos retroactivos, como evalúan los profesionales en activo la contribución que los estudios universitarios ha ejercido en su rol profesional. Éste es un campo de aplicación académico-profesional que está despertando el intereses de distintos investigadores y, por tanto, dejamos a ellos, las conclusiones que se obtengan de esos estudios (De la Fuente, 2003).

Para el acceso a los distintos cuerpos de la función docente se requiere, en la mayoría de las ocasiones, un nivel de titulación (diplomado, licenciado, arquitecto, ingeniero, doctor, etc.), no una especialidad universitaria concreta (medicina, pedagogía, farmacia, psicología, etc.). Esto hace que podamos encontrar profesionales en el ejercicio de la orientación y de la docencia, en general, que no hayan pasado por esa fase previa de formación universitaria en la disciplina más afín a su rol profesional, impregnándose del conocimiento académico y de la metodología investigativa que mejor pueda condicionar el posterior ejercicio profesional orientador. Con este análisis no tratamos de establecer categorías profesionales de eficacia y eficiencia a nivel práctico, sino únicamente de reparar en el hecho de que la formación inicial en disciplinas más o menos afines al campo profesional que posteriormente se ejerza, puede ser una variable que condicione el ejercicio profesional en el debate que estamos planteando.

Pero una vez superados los estudios universitarios viene otra fase de miniespecialización (mini, por lo reducido en el tiempo en algunas ocasiones, que no en otras) hacia la profesión a la que se aspira.

\section{Formación académico-profesional para acceder al funcionariado}

El proceso de selección que realiza la administración educativa hace que los universitarios, una vez obtenida la titulación correspondiente, deban prepararse temporalmente para superar dicho proceso selectivo. Durante esta fase, más o menos breve, en función del momento en que se convoquen dichos procesos selectivos y del momento en el que los aspirantes los superen desde que deciden iniciarse en su preparación, se realiza un período intensivo y 
específico de capacitación profesional. La cualificación profesional quedará otorgada y reconocida, al menos a nivel teórico y administrativo, en el momento en que superen esos procesos selectivos, aunque a nivel práctico la cualificación profesional requiere de una continua actualización, ya que la orientación es un verdadero reto profesional.

Algunos aspirantes eligen autoprepararse a través del estudio de los contenidos y prácticas que en cada convocatoria se especifican. Se trata una vía igualmente válida como cualquier otra, si bien tiene el inconveniente de que la parte práctica específica tiene que estar muy bien diseñada para que el aspirante sea capaz de adquirir por sí mismo, a través de autoevaluaciones continuas, las habilidades y procedimientos necesarios que le permitan enfrentarse con éxito al examen.

Otros aspirantes eligen la modalidad de entrenamiento a través de preparadores y academias que les ofertan los conocimientos necesarios y el seguimiento guiado que les permitan adquirir, al menos, los conocimientos y habilidades específicas, para superar el procedimiento selectivo. Esta alternativa tiene la ventaja de que los preparadores suelen ser profesionales en activo y que, por tanto, su bagaje experiencial entendido como conocimiento profesional (estrategias, habilidades, secuencias en el proceso de toma de decisiones del día a día en la orientación) supone un indudable recurso activo y actualizado para transferir al aspirante ese repertorio cognitivo y procedimental para la resolución de los supuestos que se le presentan en las convocatorias. Es cierto que esta modalidad depende de la pericia, la formación y la perspectiva profesional del preparador, así como de su experiencia en las tareas de preparación y también de la persistencia del preparando para asimilar esas prácticas o flexibilizarlas en el momento del examen o de su ejercicio profesional.

Este tipo de formación si bien tiene la ventaja de concentrar, al menos en la parte experiencial, la dosis de estrategias y procedimientos necesarios para afrontar la superación de los casos prácticos, deben contribuir, además, a proporcionar un nivel de autonomía y un modelo experiencial de toma de decisiones que les permitan encarar el ejercicio de la práctica profesional. Este hecho es bastante complejo, pues cabe plantearse cuántos profesionales en activo saben razonar y explicitar, en sus procesos de toma de decisiones, cuáles son las habilidades mentales y el mapa cognitivo que les ayuda en este proceso de toma de decisiones. 
Evidentemente, existe un debate alrededor del tema de si los contenidos, fases, número y tipo de ejercicios, tiempo para la realización del examen y época del año para el desarrollo del concurso-oposición son los más adecuados, así como de si en la fase de concurso existe un relativo equilibrio entre lo que pueden aportar personas en ejercicio -interinos-, generalmente valorados como méritos de antigüedad y los méritos de los que todavía no se han iniciado en la práctica profesional. Sin duda, estos temas también son importantes, ya que, al margen de sesgos interesados, no cabe duda de su incidencia en la temática que estamos abordando del desarrollo profesional.

\section{Formación inicial para los orientadores funcionarios}

La práctica habitual de cualquier empresa, al seleccionar a sus trabajadores, incluida la administración educativa, es ofrecer a los aspirantes que han superado los procedimientos selectivos cursos o actividades de formación inicial antes del ingreso activo en el cuerpo. Es lo que se conoce como período de prácticas. Aquí debemos diferenciar dos tipos de elementos:

a) Uno, el que se establece en las convocatorias de los procedimientos selectivos que suelen incluir que los aspirantes que los superen deben someterse a un período de prácticas, en el que al final de dicho proceso deben entregar una serie de documentos, generalmente memorias de lo que han realizado en la fase de prácticas en función de las directrices que se les hayan proporcionado.

b) Dos, otro proceso, más concentrado en el tiempo, en el que los responsables directos del servicio ofrecen un período de asesoramiento más o menos guiado e intensivo.

En el primero de los casos, el tutor de prácticas suele ser otro profesional de la misma especialidad, pero es una persona que ejerce su labor profesional en otro centro y, por tanto, no es observable su actuación ni puede observar a la persona en prácticas. Por tanto, su asesoramiento, en el mejor de los casos, se reduce a facilitarle las directrices que se establecen en la convocatoria de los procedimientos selectivos para la cumplimentación final de la memoria de prácticas que debe entregar al acabar dicho período.

En el segundo de los casos, se trata de cursos intensivos y concentrados durante el período inicial de los recién ingresados en el que se ofrecen las normas de organización y funcio- 
namiento mínimas, así como la normativa, conocimientos, procedimientos y mínimas habilidades en el uso de los instrumentos imprescindibles para iniciar la andadura profesional. Así, el modelo de guía experto que deben representar los formadores en esta fase tiene que ser muy cercano a los nuevos profesionales, proporcionando no sólo la normativa y las directrices (horario, Decretos de Organización y Funcionamiento, etc.), sino también una guía tutelada, que capacite al nuevo profesional de la orientación para abstraer ese modelo experiencial en el que se basa su tutor-guía.

En ciertas ocasiones, a este proceso se le acompaña de varios días de prácticas guiadas a través de la observación de un profesional en la labor cotidiana de la orientación en el centro/s de actuación. En este sistema se representa la espontaneidad de la práctica profesional, de lo que "toque" hacer en ese momento, pero el nuevo profesional pierde la perspectiva del conjunto en el que esa actuación está representada y programada. Evidentemente, por las necesidades del servicio, este modelo no se prolonga en el tiempo lo suficiente como para que el nuevo profesional extraiga de forma completa ese modelo de toma de decisiones al que nos venimos refiriendo, ni a veces está clara en la intención del tutor-guía que la finalidad de su ayuda sea proporcionarle ese modelo. Pero, al menos, sí permite que el nuevo profesional pueda comparar, si ha tenido varios modelos -guías o tutores- a su alcance, quiénes de ellos pueden servir en lo sucesivo para prolongar ese rol de asesoramiento, aunque sea a distancia.

\section{Co-formación en la práctica profesional}

En este ámbito del desarrollo profesional y docente debemos hacer varias puntualizaciones. Los profesionales de la orientación pueden desempeñar su labor profesional en Equipos de Orientación Educativa y Psicopedagógica o bien en los Departamentos de Orientación. Este hecho condiciona en gran medida lo que es el período de adaptación de los recién ingresados en el ejercicio docente, después de pasar por las fases anteriores, por varias razones. Pero quizás la más importante sea el hecho de que en los departamentos de orientación suelen ser unipersonales, al menos en estatus profesional, salvo que por su tamaño (más de 16 unidades de ESO, por ejemplo, en Andalucía) y/o características especiales (centros de actuación educativa preferente, esto es, centros de compensatoria) exista más de un orientador en el mismo centro. Mientras que en los Equipos de Orientación Educativa y Psicopedagógica, lo normal es que haya varios profesionales del mismo estatus o categoría profesional. Esto supone que siempre hay alguien con mayor grado de experiencia que puede ofrecer resortes para 
que el período de adaptación sea más eficiente (asesoramiento en el uso de instrumentos de evaluación, prestación de materiales propios elaborados para la intervención, asesoramiento en el proceso de toma de decisiones a la hora de intervenir, facilitación de los recursos necesarios para la programación, etc.).

En cambio, en los Departamentos de Orientación, durante este período inicial, se depende más de la capacidad de iniciativa del recién ingresado, así como de los procedimientos que se le proporcionaron muy especialmente durante la fase de preparación de oposiciones, si bien siempre existen compañeros en centros cercanos con los que poder contar.

Así podemos decir que el desarrollo profesional en los Equipos está condicionado, además de por variables personales (género, edad, formación inicial, experiencias laborales previas, expectativas, motivaciones e intereses al entrar en el ejercicio de la profesión, etc.), por variables grupales tales como el número de componentes de dicho equipo, la capacidad de liderazgo de alguno de ellos, la situación administrativa de los componentes -contratados laborales, interinos, en expectativas de destino, orientadores en comisión de servicios, orientadores definitivos, etc.-, grado de cooperación y compañerismo entre ellos, etc.

Los profesionales tanto de Equipos de Orientación Educativa y Psicopedagógica como los de los Departamentos de Orientación cuentan con compañeros en las Delegaciones Provinciales de Educación que también ofrecen pautas de coordinación provincial en función de las directrices que emanen de las Direcciones Generales implicadas de las Consejerías de Educación. Estas figuras pueden contribuir a proporcionar un modelo bastante homogéneo de actuación provincial, especialmente si al perfil político que suelen poseer se les añade su bagaje profesional en el campo de actuación concreto del área que coordinan. Estas figuras mantienen actualizado su conocimiento profesional si las labores de coordinación las complementan con otras labores de actuación directa (actuación en centros), pues de lo contrario acaban perdiendo la conexión con la realidad, convirtiéndose así en figuras políticas con el recuerdo de su actuación en el momento en el que ellos estuvieron desempeñando esas tareas.

\section{Perfeccionamiento profesional contínuo}

Las administraciones educativas cuentan con planes de formación permanente del profesorado, en general, y para los orientadores, en particular. Las modalidades de formación son 
variadas y abarcan desde actividades de autoformación, formación presencial, formación a distancia -on line-, cursos con seguimiento, congresos, jornadas, encuentros, simposium, jornadas científicas, etc.

Entre las modalidades de autoformación, contamos con la denominada como grupo de trabajo. Consiste en aglutinar a un grupo de personas, generalmente del mismo perfil profesional, que se reúnen periódicamente para profundizar en tareas o temas de interés común. Los recursos con los que cuentan son la búsqueda bibliográfica y el asesoramiento mutuo y recíproco entre los componentes, si bien también se les proporciona un asesoramiento externo a través de las figuras especializadas con que cuentan los Centros de Formación del Profesorado. La finalidad esencial de estos grupos suele consistir en la elaboración y/o adaptación de materiales existentes o en el estudio de casos que proporcione pautas tangibles con las que actuar ante casos similares. Esta modalidad representa una indudable ventaja, permitiendo adaptar el trabajo del grupo a los intereses del mismo, aunque es lógico que también cuente con graves inconvenientes, por ejemplo, circunscribirse a la propia perspectiva del grupo, estar limitados por el tiempo o los recursos disponibles o el desequilibrio en el trabajo aportado por cada uno de los componentes del grupo, especialmente en grupos demasiados numerosos.

Otra de las modalidades es la de cursos presenciales. Esta es una modalidad en la que un experto sobre un tema determinado presenta en una o varias sesiones un marco conceptual explicativo del mismo o los resultados concluyentes de la investigación realizada por él u otros colegas. Esta modalidad ofrece la garantía, si el experto merece esta etiqueta, de contribuir a actualizar conocimientos académicos y, a veces, abrir los ojos a los profesionales sobre posibles vías de investigación. Pero cuenta con el inconveniente de estar la mayor parte de las veces alejada del conocimiento profesional y, en los casos en los que es conocimiento derivado de la investigación, en bastantes ocasiones son investigaciones muy controladas, prácticamente de laboratorio, que no sirven para extrapolar los resultados y la metodología a contextos ecológicos más amplios. Además, presenta otros inconvenientes como tener que compatibilizar el horario personal y el profesional con los días y horas en los que se tienen que desarrollar las sesiones planificadas.

La modalidad de cursos no-presenciales -on line- suplen el inconveniente de la incompatibilidad horaria mencionada en la variante anterior y proporcionan repertorios de ejer- 
citación con los que asimilar los contenidos propuestos. Requieren del profesional que adapte en mayor o menor medida los contenidos y habilidades propuestas a las necesidades particulares de cada profesional. Sin duda, esta modalidad está adquiriendo cada vez mayor protagonismo, ofreciendo un repertorio de actividades de formación teórico-práctico con los que contribuir al desarrollo profesional, salvando las distancias geográficas.

Ejemplo de ello son las propuestas que oferta el Plan Nacional de Tecnologías de Información y Comunicación (http://www.formacion.cnice.mecd.es) o de prácticamente todas las universidades y otras instituciones privadas. Si, además, el sistema de tutoría a distancia facilita la resolución de las dificultades que se puedan plantear, incrementa aún más el potencial formador que ya poseen.

Desde nuestra perspectiva estamos convencidos de que una modalidad poco explotada hasta el momento es la que aúna el curso de experto (como facilitador del conocimiento académico) con el seguimiento recurrente con ese mismo experto después de haber experimentado propuestas prácticas acordadas en sesiones anteriores. Esta vía contribuiría a conectar el conocimiento académico con el profesional, haciéndolo más práctico y funcional, a la par que contribuiría a verificar y/o refutar algunos conceptos y teorías en vigor. En definitiva, se trata de partir de propuestas teórico-experienciales, para contrastarlas en la realidad y generar, ampliar y modificar, si cabe, los conocimientos teóricos.

Podemos revisar otras modalidades de formación tales como Jornadas de trabajo, Encuentros, Conferencias puntuales, Simposium, Congresos, etc., si bien cada una de estas modalidades también contiene sus propios inconvenientes y ventajas. Así, las Jornadas y Encuentros tienen la finalidad de presentar y confrontar las actuaciones realizadas por personas o equipos, facilitando la puesta en común de materiales elaborados por los propios participantes a partir de sus intereses y necesidades particulares, el consenso de vías de actuación o la toma de acuerdos sobre los posibles problemas existentes con ese grupo profesional. Tienen el inconveniente de la concentración de las actividades en períodos muy cortos (un par de días) y, a la vez, espaciados en el tiempo (final de cada curso).

Los ciclos de Conferencias tienen la ventaja de presentar tópicos muy concretos a lo largo de un período de tiempo, si bien no permiten profundizar en esos mismos aspectos. En 
cualquier caso siempre cabe la posibilidad, en un futuro cercano, de poder establecer contactos con esos expertos para realizar otras modalidades de formación.

Los Congresos, en función de su ámbito de reconocimiento, representan una oportunidad para recepcionar distintos marcos teóricos sobre aspectos del conocimiento profesional. Pero además presentan la ventaja de poder exponer las experiencias desarrolladas por los profesionales a pie de obra, a través de comunicaciones y póster. Sin embargo, cuentan con graves inconvenientes, tales como el alto costo de la matrícula, al que hay que sumar los gastos de manutención y alojamiento, junto con las dificultades para la asistencia cuando se desarrollan en lugares lejanos y/o en horario y tiempo laboral.

\section{Perfeccionamiento académico-profesional recurrente}

La administración educativa está ofreciendo la posibilidad de seguir formándose de forma recurrente a través de la modalidad de Licencia por Estudios. Consiste básicamente en proporcionar períodos de tiempo (tres, seis meses o un año) remunerados con la posibilidad de estar total o parcialmente exento de las obligaciones laborales. Aquí, los profesionales pueden seguir adquiriendo más conocimiento académico habiendo desarrollado previamente conocimientos de tipo profesional. Por ello, el profesional tiene que reelaborar sus propias concepciones teóricas a partir de los supuestos experienciales adquiridos en etapas anteriores. Por ello, esta modalidad permite funcionalizar la teoría y racionalizar, hacer más explicativa la práctica. Si bien es una vía interesante, la oferta es claramente insuficiente para lo que la demanda requiere.

Hasta aquí hemos hecho una reflexión por escrito de cómo el conocimiento académico puede contribuir desde unos orígenes muy cercanos a la vida activa y laboral (los estudios universitarios) al desarrollo profesional, hasta otras modalidades de formación que repercuten en la misma línea.

Llegados a este punto debemos plantearnos cómo se diseña desde la legislación vigente la actuación en orientación y cómo valoran los organismos de control y evaluación de la enseñanza las actuaciones en orientación o la percepción que de la orientación tienen los usuarios y receptores directos (alumnado). 


\section{Contribución de la legislación vigente al desarrollo del perfil de orientador}

Algunas de las tareas y funciones que se recogen en las legislación vigente Ley Orgánica 10/2002, de 23 de diciembre, de Calidad de la Educación (BOE 24-12-2002) nos lleva a preguntarnos qué panorama se nos presenta ante la nueva ley en cuanto a la homogeneización de las actuaciones. Sabiendo que toda Ley Orgánica debe desarrollarse posteriormente, podemos obervar un plantemiento de la actuación orientadora amplio. Algunas especificaciones que hace la ley en este aspecto son:

- Se prestará especial atención en la Educación Primaria a la atención individualizada de los alumnos, la realización de diagnósticos precoces y el establecimiento de mecanismos de refuerzo para evitar el fracaso escolar (art. 16.4).

- Emitir informes de orientación escolar para cada alumno (al término de $2^{\circ}$ de ESO, art. 26.3). El equipo de evaluación estará asesorado por el equipo de orientación.

- Incidencia en la orientación educativa y profesional en los Programas de Iniciación Profesional (art. 27; especialmente el 27.4).

- Asesoramiento a otros profesionales que prestan sus servicios a alumnos con necesidades educativas especiales (ESO, art. 32.4).

- Análisis y valoración de necesidades educativas (art. 45.2), por equipos de profesionales con distintas cualificaciones.

- El profesorado colaborará con los servicios o departamentos especializados en orientación, en el proceso de orientación educativa, académica y profesional del alumnado (art. 56.e).

- Funciones de asesoramiento en el centro, mediante los órganos de coordinación didáctica (art. 85.1) o el establecimiento de otros órganos de coordinación por parte de la administración (art. 85.2).

En definitiva, el panorama que se dibuja es tan amplio que caben multitud de actuaciones. Ante las mismas, cada profesional puede actuar de múltiples modos, a veces complementarios y, a veces, contrapuestos.

\section{¿Qué consideración tiene el sistema educativo y sus usuarios sobre el rol de la orienta- ción?}


El Servicio de Inspección Educativa en Madrid capital, referente al curso 1999-2000, concluye varios hechos importantes en la etapa de Educación Primaria:

- Dos tercios de los centros valorados admiten una valoración aceptablemente positiva de la acción tutorial y orientadora.

- Existe una notable diferencia entre centros y maestros al comparar las tareas de planificación y ejecución de la orientación y la tutoría de los alumnos.

- Como aspectos positivos se destacan:

- Adecuada actuación con las familias a través de las entrevistas individualizadas.

- Acertadas actuaciones de orientación y atención a los alumnos con problemas de aprendizaje.

- Planificación y desarrollo de actuaciones de acogida para nuevos alumnos.

- Incremento de actitudes de participación, tolerancia y solidaridad entre los alumnos.

- Pertinente registro de la evaluación continua, aunque gran diferencia en la sistematización de los mismos.

○ Buena coordinación del profesor-tutor con el resto de maestros que intervienen con el grupo de alumnos.

- En cuanto a los aspectos que deben mejorarse se señalan:

- Escaso desarrollo del Pan de Orientación y Acción Tutorial en los documentos de planificación a medio y corto plazo del centro (Proyecto Educativo y Proyecto Curricular).

- Planes de Acción Tutorial demasiado genéricos y poco operativos para los tutores.

- Escasa atención a los alumnos con sobredotación intelectual.

○ Confección de Informes Individualizados con datos demasiados generales que con frecuencia no expresan las medidas específicas de refuerzo o adaptación curricular.

- Deficiente coordinación de las tareas de orientación y acción tutorial por parte del Claustro de Profesores, la Comisión de Coordinación Pedagógica e incluso la Jefatura de Estudios. Por lo que la actuación de los tutores se deja llevar por criterios personales. 
○ La participación del orientador de los equipos en la elaboración, desarrollo y revisión del Plan de Acción Tutorial y de la organización de la orientación educativa es dispar entre los diferentes centros.

Respecto a las conclusiones que se hacen referentes a los datos obtenidos durante el curso académico 1999-2000, en Madrid capital, sobre la tutoría y la orientación académica profesional en la Enseñanza Secundaria, se señalan los siguientes indicadores:

- El estado de la tutoría y de la orientación académica y profesional no parece del todo insatisfactorio, si bien se observa una evidente mejora desde una situación inicial de falta de tradición y fuertes resistencias de una parte del profesorado (y, en ocasiones, también del alumnado).

- Los aspectos en los que se señalan que se debe mejorar son:

- Entre una cuarta parte y un tercio de los centros no se han alcanzado las condiciones necesarias para un correcto desarrollo. En otro grupo de centros, aproximadamente entre un 10 o $15 \%$, se ha progresado en los aspectos formales, pero falta por consolidar aspectos importantes.

- Más de la mitad de los centros (60\%) se dan las condiciones adecuadas tanto en documentos de programación como en los aspectos organizativos de la tutoría, para que ésta se desarrolle de forma adecuada, aunque perfeccionable.

- Como aspectos positivos se señalan:

- Casi la totalidad de los centros disponen, según la norma, del Plan de Orientación Académico y Profesional y de Plan de Acción Tutorial, integrados en el Proyecto Curricular.

- Los aspectos formales y las exigencias normativas básicas de la tutoría se encuentran aseguradas.

- Las actividades dirigidas a los alumnos que mejor consolidadas están son las destinadas a la acogida e integración en el centro, pero especialmente las relacionadas con el compromiso y asunción de las normas de convivencia (disciplina que se reflejará en los Reglamentos de Organización y Funcionamiento de los Centros).

○ La provisión de recursos bibliográficos y documentos de apoyo al profesorado, relacionados con la tutoría y la orientación, se consideran muy satisfactorias. 
- Como aspectos negativos se señalan:

○ La incongruencia entre los planteamientos efectuados en el Proyecto Educativo y el Proyecto Curricular.

- La escasa participación y protagonismo de la Comisión de Coordinación Pedagógica en la elaboración de las directrices para el Plan de Acción Tutorial y el Plan de Orientación Académica y Profesional, así como casi la nula implicación del Claustro en la coordinación de la acción tutorial.

- Desconexión con el tejido productivo y la vida laboral activa, pocas directrices para las familias y para los departamentos didácticos en relación al proceso de enseñanza-aprendizaje.

- Inexistencia de directrices y criterios para evaluar el propio Plan.

- Los déficit más acusados corresponden al ámbito de la coordinación:

- Solo en algo más de la mitad de los centros se realizan las reuniones entre la Jefatura de Estudios y/o orientador con los tutores.

- Las reuniones periódicas de los equipos educativos para coordinar el proceso de aprendizaje, promovidas por el tutor, escasean.

- La coordinación para la evaluación continua a lo largo de todo el curso, función primordial del tutor, se limita, en la mayor parte de las ocasiones a las sesiones de evaluación preceptivas.

- Las entrevistas individuales con los alumnos para determinar aspectos negativos y positivos de su proceso de aprendizaje se reducen a casos de indisciplina y mal comportamiento.

- Las reuniones colectivas de los tutores con las familias del alumnado aparece como otro de los aspectos débiles de la acción tutorial.

- Más de un tercio de los centros el Jefe del Departamento de Orientación no incluye, según es preceptivo, en su horario personal dos tardes a la semana para la atención a padres y orientación de alumnos. Los pocos que cumplen esta norma se reducen a una semana.

- Existen notables diferencias en cuanto a la atención que recibe la orientación y la acción tutorial en Secundaria Obligatoria, especialmente en el primer ciclo, en comparación con el Bachillerato. Esto es así hasta tal punto que de los pocos centros que se les ha autorizado a que en $2^{\circ}$ de Bachillerato planifiquen una hora de tutoría lectiva, éstos la dedican a dar clase. 
Si bien, las conclusiones que arroja este informe parecen concluyentes, debemos considerar otras que la investigación reciente señala acerca de percepción que los usuarios tienen de las actividades de orientación.

Interpretación de los usuarios directos sobre las actividades de orientación

La lectura reciente de la tesis doctoral de Fernández (2003) puede darnos idea de cómo perciben y valoran el alumnado y el profesorado las actividades de orientación y tutoría.

- Percepción del profesorado:

○ La actuación de los profesionales de los equipos es percibida por los maestros de Educación Infantil y Educación Primaria como reactiva, individual y centrada exclusivamente en temáticas relacionadas con el proceso de enseñanza-aprendizaje.

○ La intervención directa de los orientadores en casos de conflictividad y problemas de convivencia se realiza de forma reactiva, individual y mediante un asesoramiento basado en el modelo de experto.

- La intervención preactiva se asocia a la acción tutorial y orientación de los profesionales de los departamentos.

- Los receptores y usuarios directos de la orientación han evolucionado hacia una consideración mayor del modelo colaborativo de trabajo de los orientadores.

- La mayor eficacia de la orientación ejercida desde los centros es una realidad que se detecta a lo largo de todo el estudio.

- Los estudiantes piden más atención directa por parte de los profesionales de la orientación. Esperan de ellos un trato más respetuoso, más en el ámbito de la amistad, buscando un adulto de confianza.

- El profesorado de Primaria y Secundaria estiman la conveniencia de incrementar el número de profesionales en los centros y equipos, si bien han de aclarar su Plan de Trabajo (explicando qué van a hacer y cómo) e incrementar el contacto directo con alumnado y profesorado.

- Este mismo profesional de la orientación (Fernández, 2003) establece el perfil ideal de la figura del orientador:

- Capacidad de comunicación y escucha, empatía, carácter abierto, que transmita credibilidad y confianza. 
○ Implicación en los problemas del centro, compromiso, integración en el centro.

- Accesible, disponible, flexible a la hora de reunirse, buen trato, amable, optimista, con espíritu de servicio, cercano, con capacidad de iniciativa, dinamizador y responsable.

- Honestidad profesional, sinceridad, profesionalidad, coherencia, constancia, actitud positiva.

- Cualificación profesional, actitud permanente de formación, tener un buen marco teórico y conocimiento del sistema educativo.

\section{Nuevos retos en el futuro de la profesión orientadora}

El desarrollo de las tareas orientadoras es una profesión fascinante para aquellos que la ejercemos. El esbozo que hemos realizado de las bondades y limitaciones del desarrollo profesional a través del influjo de la formación y el perfeccionamiento nos debería llevar a establecer nuevos planteamientos que los optimicen.

A nivel profesional debemos tender a la convergencia. En este sentido debemos mencionar las numerosas asociaciones que están surgiendo en las distintas comunidades autónomas, con intereses y finalidades variados, pero con puntos de confluencia, en todas ellas sus profesionales intervienen en el ámbito de la orientación. A nivel supraorganizacional está la iniciativa que surgió en el Congreso Internacional de Orientación y Tutoría, en Zaragoza, (junio de 2003), con el ánimo de aglutinar a las numerosas asociaciones nacionales. Directivos y compañeros de la Asociación Aragonesa de Psicopedagogía tuvieron iniciativas muy brillantes encaminadas a darle sentido y forma esta idea. Así surgió la COPOE, la Confederación de Organizaciones de la Psicopedagogía y la Orientación de España. Su constitución formal ya tiene fecha formal el 12 de marzo de 2005, coincidiendo con Aula, el Salón del Estudiante y la Oferta Educativa, donde los distintos representantes de las asociaciones, federaciones o secciones de colegios oficiales podrán formalizar su adhesión a esta confederación, que tendrá, entre otros objetivos, los de representar los intereses, fines y posicionamientos de las organizaciones que compongan la confederación, pero también los de sensibilizar a la sociedad de la importancia de las funciones de la orientación, la consulta y la investigación para el desarrollo integral de las personas (Escuela Española, 30-09-2004). 
Así pues, la definición y el avance de nuestro perfil profesional pasa por canalizar nuestras iniciativas en las asociaciones de ámbito más restringido, pero también es ésta de ámbito más extenso, de modo que sirvan para hacer llegar nuestras inquietudes, propuestas a los gobiernos y poder converger en el ámbito europeo en una entidad suficientemente representativa.

En el documento a debate del Ministerio de Educación, titulado "Una educación de calidad para todos y entre todos" mostramos la sensibilidad ante la ausencia de referencias a los temas de orientación y tutoría, en cuanto derecho que debe ser para los alumnos estar asistidos en su orientación académica, vocacional y profesional, así como la necesaria potenciación de los Departamentos de Orientación incrementando los recursos humanos y materiales con que cuentan.

No podemos pasar por alto que vivimos en la era de las tecnologías de la información y la comunicación, que han invadido todos los sectores productivos y profesionales, hasta nuestras vidas y nuestro ocio personal. El rol del orientador debe contemplar su actualización en este aspecto, a la par que su intervención (análisis, evaluación e intervención) también pueden estar condicionadas por el espectro tecnológico.

La orientación educativa está abocada, como una parte más del fenómeno educativo, a entenderse y coordinarse de forma interintitucional con otros entes. En unas ocasiones, para desarrollar tareas complementarias, por ejemplo entre orientadores de secundaria (etapa educativa que puede tener un carácter terminal, por lo que habrá alumnos que decidan incorporarse a la vida activa y laboral) y otros orientadores, por ejemplo, los de Andalucía Orienta u otros sectores -fuerzas armadas, gabinetes de selección de recursos humanos, etc. En otras ocasiones, la cooperación con el propio mundo universitario, de forma que podamos contribuir por una parte, de forma copartícipe -entre académicos y profesionales-, a hacer más funcional la teoría psicopedagógica y, por otra, a racionalizar y desarrollar la práctica profesional. En pocas palabras, a la cooperación de investigación y desarrollo en las tareas de orientación, tutoría, psicopedagogía y/o psicología educativa. 


\section{Referencias}

Bisquerra, R. (2004). Orientación y Tutoría. En J.L. Soler Nages; Orientación y Tutoría. (pp. 66-77). Zaragoza: Mira Editores.

De la Fuente, J. (2003). ¿Por qué los alumnos no construyen un conocimiento psicológico académico y profesional integrado? Reflexiones para una investigación necesaria. $P a-$ peles del Psicólogo, 24 (86), 34-41.

Fernández, J. de D. (2003). Los Equipos y los Departamentos de Orientación: Caves para el desarrollo de sus profesionales. Universidad de Granada. Tesis Doctoral.

Inspección de Educación (s/f): La Tutoría y la Orientación Académica y Profesional. Educación Secundaria. Informe de Supervisión. Curso 1999-2000. Comunidad de Madrid: Consejería de Educación.

Inspección de Educación (s/f): La Tutoría y la Orientación Educativa del Alumnado de Educación Primaria. Informe de Supervisión. Curso 1999-2000. Comunidad de Madrid: Consejería de Educación. 
[Página dejada en blanco intencionadamente] 